\title{
A shape visualization of a magnetic anisotropy energy density of single-domain magnetic nanoparticles
}

\author{
R.A Rytovi,A,B, N.A. Usov²,A \\ A National Research Nuclear University "MEPhI" \\ B National Research Technological University "MISIS" \\ ${ }_{1}$ ORCID: 00o0-0002-7963-3673, ruslan.rytov2017@yandex.ru \\ 2 ORCID: 0000-0002-2061-3467, usov@obninsk.ru
}

\begin{abstract}
$\underline{\text { Abstract }}$
A key physical parameter determining stationary directions of a magnetic moment of a single-domain ferromagnetic nanoparticle is a type of its effective magnetic anisotropy. Stationary directions of a magnetic moment of a particle change under an influence of an external magnetic field. For better understanding of a behavior of a magnetic moment of a nanoparticle in an external magnetic field, we proposed a simple method for visualization of an energy density of magnetic anisotropy of a single-domain magnetic nanoparticle. In a spherical coordinate system, an energy density of magnetic anisotropy is represented as a certain surface, which makes it possible to clearly demonstrate the presence of energy minima that determine the equilibrium directions of a single vector of magnetization of a nanoparticle in space. The cases of uniaxial, cubic, and combined magnetic anisotropy are considered in detail. The change in the total energy of a magnetic nanoparticle under the influence of an external uniform magnetic field is demonstrated.
\end{abstract}

Keywords: magnetic anisotropy, magnetic nanoparticles, visualization, energy density, magnetic field.

\section{Introduction}

Magnetic nanoparticles are widely used in modern technologies, such as magnetic recording of high density information, production of permanent magnets and magnetic fluids, microelectronics, etc. $[1,2]$. Recently, ensembles of magnetic nanoparticles have been used with great success in biomedicine, magnetic resonance imaging, targeted drug delivery, purification of biological media from toxins, magnetic hyperthermia, to fight malignant neoplasms, and in many other areas [3-6].

Meanwhile, physics of ensembles of magnetic nanoparticles is very complex [7-11]. The physical properties of an ensemble are determined by a large combination of geometric and magnetic parameters, such as particle size and shape, saturation magnetization, type of magnetic anisotropy, and values of magnetic anisotropy constants. An ensemble density, viscosity, and temperature of the medium in which magnetic nanoparticles are distributed are also of great importance. For better understanding a complex behavior of an ensemble of magnetic nanoparticles under various conditions, it is useful to first study an evolution of a magnetic moment of an isolated single-domain magnetic nanoparticle when an external magnetic field changes. This behavior substantially depends on the type of effective magnetic anisotropy of the nanoparticle [7-9].

Visualization of complex physical processes is often used as one of the ways to study phenomena occurring at the atomic level $[12,13]$. For example visualization method is used for studies of physical processes in nanostructures in [12]. Visualization of complex, multidimensional numerical data allows us to understand the processes taking place in the studied objects. 
Therefore, a graphic representation of the various types of magnetic anisotropy that exist in magnetic nanoparticles makes it easier for students and young researchers to understand the essence of complex physical processes.

This article is devoted to the development of a graphical representation of an energy density of magnetic anisotropy of single-domain magnetic nanoparticles. A change in the total energy of a magnetic nanoparticle under the influence of an external uniform magnetic field is also considered. All images shown in this article were obtained using Matplotlib data visualization package in Python programming language.

\section{Properties of magnetic nanoparticles}

As is known [7-9], in a ferromagnetic sample, a modulus of a local magnetization vector $\vec{M}(\vec{r})$ is constant and equal to the saturation magnetization of a ferromagnet, $|\vec{M}(\vec{r})|=M_{s}$.

The direction of this vector is described by a unit magnetization vector, $\vec{\alpha}(\vec{r})=\vec{M}(\vec{r}) / M_{s}$ $|\vec{\alpha}(\vec{r})|=1$. In most cases, single-domain magnetic nanoparticles, which size is smaller than the diameter of a single-domain particle $D<D_{c}$, are most interesting for applications. A singledomain nanoparticle in the lowest energy state is uniformly magnetized. Such a particle is a small permanent magnet, which creates a sufficiently strong inhomogeneous magnetic field in the surrounding space. The density of magnetic energy of a uniformly magnetized particle is high, because it is proportional to [7-9] the square of the saturation magnetization, $w_{m} \sim$ $M_{s}{ }^{2}$. However, for a single-domain spherical nanoparticle, a magnetic energy density does not depend on the direction of a single magnetization vector due to symmetry in a distribution of magnetic charges on the surface of the sphere. At the same time, experiment shows that in equilibrium, in absence of an external magnetic field, the unit magnetization vector of a single-domain spherical nanoparticle is oriented in strictly defined directions with respect to the symmetry axes of the crystal lattice of the particle. These distinguished spatial directions are called axes of easy magnetization of a nanoparticle (also, easy axes of anisotropy of a particle). Since an exchange energy of a uniformly magnetized particle is equal to zero, and a magnetic energy of a spherical nanoparticle does not depend on the direction of a single magnetization vector, the directions of easy anisotropy axes are determined by the form of the energy density of magnetic crystallographic anisotropy, $w_{a}=w_{a}(\vec{\alpha})$ [7-11].

For crystals with a single axis of symmetry, it follows from general considerations [7-11] that the energy density of magnetic crystallographic anisotropy can be represented as an expansion in powers of a unit magnetization vector

$$
w_{a}(\vec{\alpha})=K_{1}\left(\alpha_{x}^{2}+\alpha_{y}^{2}\right)+K_{2}\left(\alpha_{x}^{2}+\alpha_{y}^{2}\right)^{2}+\ldots,
$$

where, for simplicity, it is assumed that selected axis of symmetry of a crystal is parallel to the $\mathrm{z}$ axis of a Cartesian coordinate system. In equation (1), $K_{1}, K_{2}$, etc. there are uniaxial magnetic anisotropy constants, which usually decrease in absolute value, $\left|K_{1}\right|>\left|K_{2}\right|$. From equation (1) it follows that in the case $K_{1}, K_{2}>0$ an energy density of uniaxial magnetic anisotropy has the smallest value, $w_{a}=0$, if the unit magnetization vector is parallel to the $\mathrm{z}$ axis, i.e $\vec{\alpha}=(0,0,1)$ or $\vec{\alpha}=(0,0,-1)$. Thus, for a particle with anisotropy energy density (1), these directions of the unit vector are preferable.

On the other hand, from symmetry considerations it follows [7-11] that for crystals with cubic symmetry of the crystal lattice, an energy density of magnetic crystallographic anisotropy can be specified as

$$
w_{a}(\vec{\alpha})=K_{1 c}\left(\alpha_{x}^{2} \alpha_{y}^{2}+\alpha_{x}^{2} \alpha_{z}^{2}+\alpha_{y}^{2} \alpha_{z}^{2}\right)+K_{2 c} \alpha_{x}^{2} \alpha_{y}^{2} \alpha_{z}^{2}+\ldots,
$$

when $K_{1 \mathrm{c}}>\mathrm{O}, K_{2 \mathrm{c}} \approx \mathrm{o}$ the directions of easy magnetization of a particle with cubic anisotropy are parallel to axes of a Cartesian coordinate system, since the magnetic anisotropy energy (2) 
is minimal, $w_{a}=\mathrm{o}$, when the unit magnetization vector is parallel to the $\mathrm{x}, \mathrm{y}$, or $\mathrm{z}$ axes, i.e. $\vec{\alpha}=( \pm 1,0,0)$, etc. Thus, in this case, a particle has 6 free easy magnetization. If a constant $K_{1 \mathrm{c}}<$ $\mathrm{o}$, then directions of easy magnetization are parallel to diagonals of a cube, and the magnetic anisotropy energy (2) in this case has a minimum for the vector form $\vec{\alpha}=( \pm 1 / \sqrt{3}, \pm 1 / \sqrt{3}, \pm 1 / \sqrt{3})$.

It should be noted, however, that expressions (1), (2) are only the simplest energy contributions that determine a direction of the axes of easy magnetization of a nanoparticle in space. Another important contribution to the effective magnetic anisotropy is related to the difference between the shape of the particle and the spherical one. This contribution is called the shape anisotropy energy [7-9]. It is especially important for magnetically soft type nanoparticles with a sufficiently high saturation magnetization. A famous Braun - Morrish theorem [12] claims that a magnetostatic energy of a uniformly magnetized particle of arbitrary shape, to a first approximation, coincides with a magnetostatic energy of some equivalent ellipsoid. If axes of a Cartesian coordinate system are chosen along the symmetry axes of the equivalent ellipsoid, then a magnetic energy density of a nanoparticle will take the form [14]

$$
w_{m}(\vec{\alpha})=\frac{1}{2} M_{s}^{2}\left(N_{x} \alpha_{x}^{2}+N_{y} \alpha_{y}^{2}+N_{z} \alpha_{z}^{2}\right),
$$

where $N_{x}, N_{y}, N_{z}$ are demagnetizing factors of an equivalent ellipsoid in an indicated coordinate system.

Obviously, demagnetizing factors of a cube-shaped particle are equal to each other due to symmetry, $N_{x}=N_{y}=N_{z}=4 \pi / 3$. Given that $N_{x}=N_{y}=N_{z}=4 \pi / 3$, it is easy to see that the magnetic energy of a uniformly magnetized cube, like the magnetic energy of a spherical nanoparticle, does not depend on the direction of a single magnetization vector and does not contribute to the magnetic anisotropy of the particle. On the other hand, for a typical case of an elongated spheroid, for which the transverse demagnetizing factors are equal, $N_{x}=N_{y}>$ $N_{z}$, the magnetic energy of the particle (3) can be written as

$$
w_{m}(\vec{\alpha})=\frac{1}{2} M_{s}^{2}\left(N_{x}-N_{z}\right)\left(\alpha_{x}^{2}+\alpha_{y}^{2}\right)+\text { const }
$$

We note here that an addition of an arbitrary constant to an energy density of magnetic anisotropy obviously does not affect the direction of the light axes of the particle anisotropy, and does not affect the dynamics of the unit magnetization vector [7-9]. This situation is similar to a case of classical mechanics, where the potential energy of a particle is given up to an arbitrary constant, because a force acting on the particle is determined by a gradient of potential energy.

Comparing equations (1) and (4), we can conclude that an elongated equivalent spheroid can be characterized by an effective shape anisotropy constant, $K_{e f}=M_{s}^{2}\left(N_{x}-N_{z}\right) / 2$. Moreover, both the direction of the easy axis of anisotropy and the values of the effective demagnetizing factors $N_{x}$ and $N_{z}$ are determined [14] by the real shape of the non-ellipsoidal nanoparticle. A total energy of a magnetic anisotropy of a particle in a general case is the sum of an energy of magnetocrystallographic anisotropy and a shape anisotropy energy. In this case, it takes place [15] an effective combined magnetic anisotropy of a nanoparticle, $w_{e f}(\vec{\alpha})$.

In a presence of a uniform external magnetic field $\boldsymbol{H}$, a single-domain nanoparticle remains uniformly magnetized. In this case, stationary, time-independent, directions of a unit magnetization vector are determined by a minima of a total energy of a particle in an external magnetic field

$$
w_{t}(\vec{\alpha})=w_{e f}(\vec{\alpha})-M_{s} \vec{H} \vec{\alpha},
$$


Naturally, a position of minima and maxima of a total energy of a single-domain nanoparticle and their number changes with a change in a magnitude and direction of the external magnetic field with respect to direction of easy axes of anisotropy of a particle.

\section{Visualization of an energy density of a particle anisot- ropy}

\subsection{Uniaxial and cubic types of magnetic anisotropy}

In order to clearly demonstrate a location of minima and maxima of an energy density of magnetic anisotropy, it is convenient to set this energy in a spherical coordinate system [7], $w_{a}=w_{a}(\theta, \varphi)$, by expressing in equations (1) - (5) components of a unit magnetization vector through spherical angles $(\theta, \varphi)$, so that $\alpha_{x}=\sin \theta \cos \varphi, \alpha_{y}=\sin \theta \sin \varphi, \alpha_{z}=\cos \theta$. Then in a spherical coordinate system it is possible to construct a certain surface of a form $r(\theta, \varphi)=w_{a}(\theta, \varphi)$.
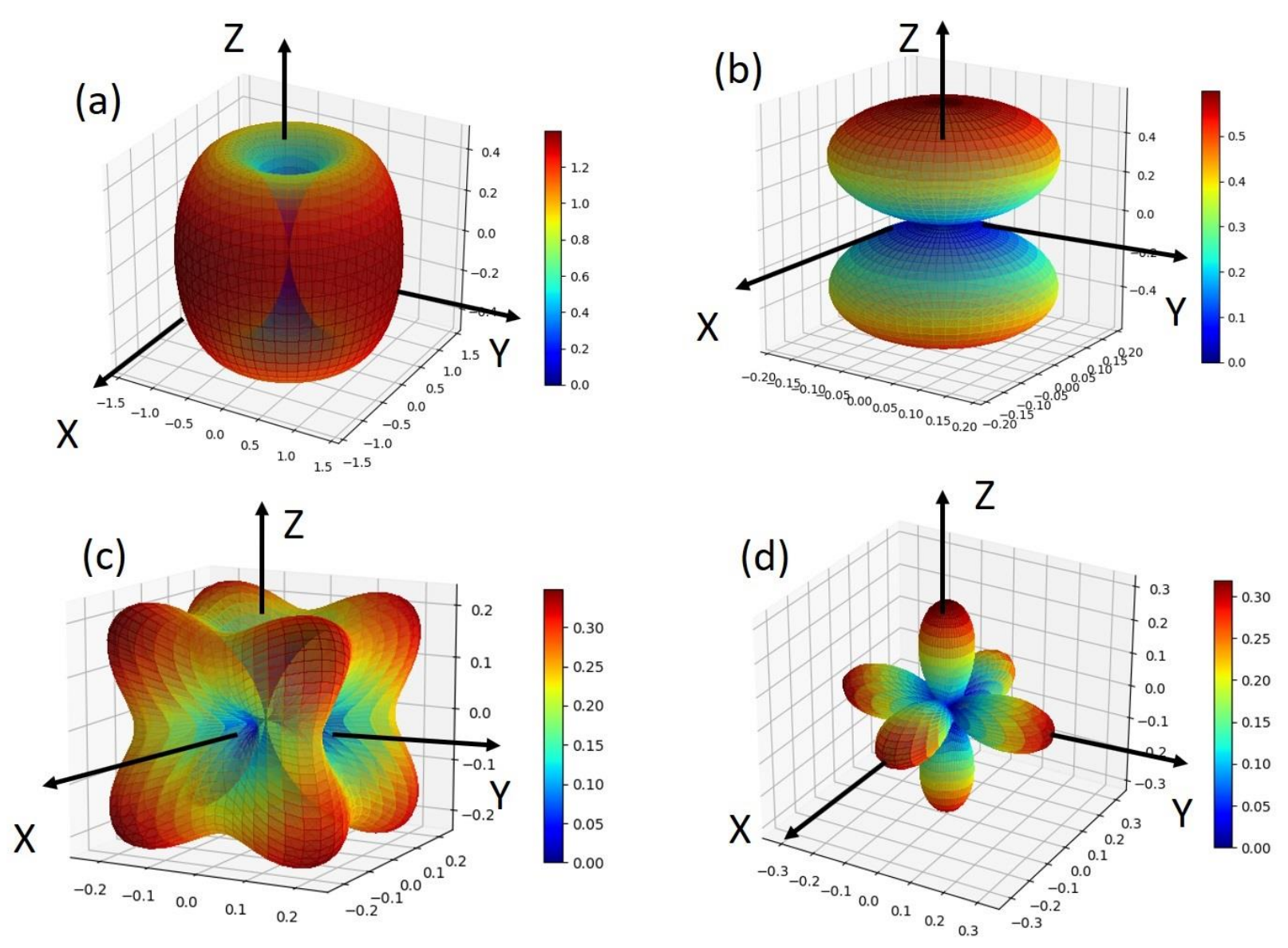

Fig. 1. An energy density of magnetic anisotropy for uniaxial and cubic types of magnetic anisotropy of a nanoparticle.

Figure 1a shows a reduced energy density of uniaxial magnetic anisotropy, $w_{a}(\theta, \varphi) / K_{1}$, equation (1), for a case $K_{1}>0, K_{2}=0$. Obviously, an energy density of magnetic anisotropy has deep minima for directions of a unit magnetization vector close to the angles $\theta=0$, $\pi$. In this case, it is said that a particle has two deep potential wells separated by a high symmetric potential barrier, which maximum corresponds to the angle $\theta=\pi / 2$. 
Figure $1 \mathrm{~b}$ shows a reduced energy density of uniaxial magnetic anisotropy (1) for a case $K_{1}<$ $\mathrm{o}, K_{2}=0$. To exclude negative radius values in a dependence $r=w_{a}(\theta, \varphi)$, a corresponding positive constant is added to equation (1). As Fig. $1 \mathrm{~b}$, in a case under consideration, a minimum energy corresponds to directions lying in a plane $\theta=\pi / 2$. This type of anisotropy is called a "easy plane" because an anisotropy energy remains minimal, equal to zero, for all directions lying in this plane. Real particles can have additional weak contributions to an energy of magnetic anisotropy, which emit some directions in an indicated plane, separated, generally speaking, by small energy barriers.

In Fig. 1c shows a reduced energy density of cubic magnetic anisotropy, $w_{a}(\theta, \varphi) / K_{1 c}$, equation (2), for a case $K_{1 \mathrm{c}}>\mathrm{O}, K_{2 \mathrm{c}}=0$. In this case, an anisotropy energy minima correspond to directions parallel to axes of a Cartesian coordinate system. In total, there are thus 6 energy minima, in other words, deep potential wells separated by energy barriers. In an ideal case of a spherical nanoparticle, all these potential wells are completely equivalent, so that in equilibrium a magnetic moment of a particle can be directed in any of an indicated directions of easy magnetization. At the same time, for a case $K_{1 \mathrm{c}}<0, K_{2 \mathrm{c}}=0$, shown in Fig. $1 \mathrm{~d}$, a minimum energy corresponds to directions parallel to diagonals of a cube. Therefore, in this case, there are 8 equivalent directions for which a magnetic anisotropy energy of a particle has a minimum.

\subsection{Combined type of magnetic anisotropy}

Energy surfaces shown in Fig. 1a - 1d correspond to an ideal case of a spherical magnetic nanoparticle. If a shape of a particle deviates from spherical, then an energy of magnetic anisotropy, equations (1) - (3), is added to an energy of a shape anisotropy, equation (4), which can make a significant contribution to a total anisotropy energy for particles with high saturation magnetization.

(a)
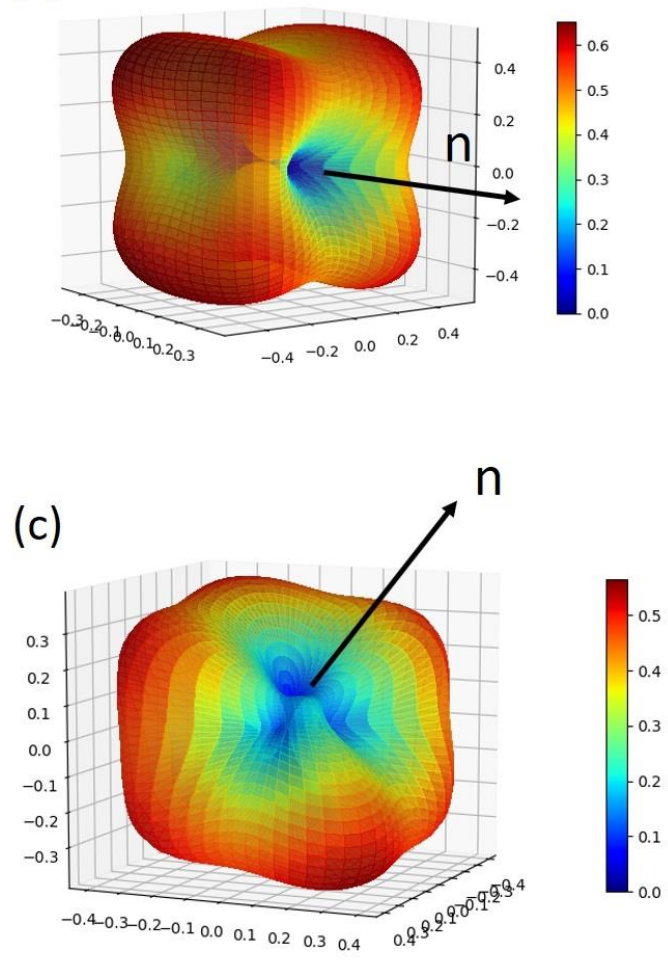

(b)
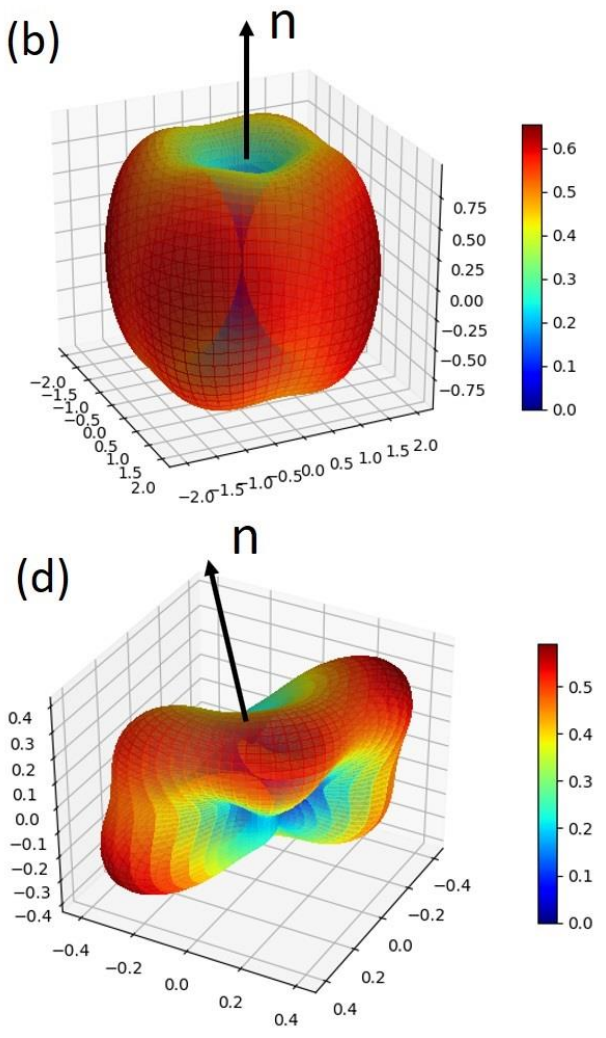

Fig. 2. An energy density for a combined type of magnetic anisotropy of a particle (cubic anisotropy + uniaxial shape anisotropy). 
As an example, Figure 2 shows the case of combined magnetic anisotropy. Here to the energy density of cubic anisotropy, $w_{a}(\theta, \varphi) / K_{1 c}$, shown in Fig. 1c, a reduced energy density of the shape anisotropy, $K_{e f}\left(1-(\vec{\alpha} \vec{n})^{2}\right) / K_{1 c}$, is added. A unit vector $\boldsymbol{n}$ indicates a direction of the easy axis of a shape anisotropy.

First, we consider a simple case when the axis of a shape anisotropy energy coincides with one of the axes of cubic anisotropy (see Fig. 2a, 2b). In Fig. 2a, a ratio of anisotropy constants was chosen small, $K_{e f} / K_{1 c}=0.1$. However, as shown in Fig. 2a, due to an influence of a shape anisotropy energy, a surface of a combined anisotropy energy becomes, in fact, two-pit already for small values of the $K_{e f} / K_{1 c}$ ratio, and two main, deep potential wells are located in directions parallel to the vector $\boldsymbol{n}$. In this case, lateral energy minima are easily distinguishable in Fig. 2a, they disappear only at $K_{e f} / K_{1 c} \geq 1$.0. So, Figure $2 b$ shows a case of $K_{e f} / K_{1 c}=2.0$, when only two energy minima remain in directions parallel to the vector $\boldsymbol{n}$.

If a vector $\boldsymbol{n}$ is oriented arbitrarily with respect to cubic axes of anisotropy (see Fig. 2c, 2d), then a corresponding energy surface becomes very complex for sufficiently small values of a ratio $K_{e f} / K_{1 c}$. However, with an increase in this ratio, $K_{e f} / K_{1 c}=0.1$, a global energy surface also becomes two-fold, as Fig. 2c, $2 \mathrm{~d}$ for the directions of the vector $\boldsymbol{n}$ given by angles $(\theta=\pi / 4$, $\varphi=\pi / 4)$ и $(\theta=-\pi / 4, \varphi=\pi / 4)$, respectively. Note that a barrier separating potential wells has a very complex shape. A shape of an energy barrier between potential wells is extremely important because it determines a position of an energy saddles, or passes that connect adjacent potential wells. At finite temperatures, due to thermal fluctuations, a magnetic moment of a nanoparticle can jump between different potential wells, and such jumps in a magnetic moment are most likely to occur through the saddle regions of the energy barrier.

\subsection{An influence of an external magnetic field}

As discussed above, taking into account a magnetic anisotropy energy, a vector of a magnetic moment of a nanoparticle has several stable directions in space for which a total energy of a nanoparticle has a local minimum. In an absence of an external magnetic field, neglecting thermal fluctuations, a magnetic moment of a single-domain nanoparticle is in one of the energy minima. In an applied external magnetic field, the energy minima of potential wells shift with respect to each other, some minima disappear, others appear. Therefore, when an external magnetic field changes, a magnetic moment of a particle can jump from one potential well to another. In Fig. 3a-3d shows an example of a change in a total energy density of a uniaxial nanoparticle,

$$
\frac{w_{t}(\vec{\alpha})}{K_{1}}=\alpha_{x}^{2}+\alpha_{y}^{2}-2 \vec{h} \vec{\alpha}
$$

under an influence of an external magnetic field, where the magnitude of a magnetic field is normalized to the particle anisotropy field, $\vec{h}=\vec{H} / H_{a}, H_{a}=2 K_{1} / M_{s}$. A magnitude of a magnetic field in Fig. 3a-3d is equal to $H=0,300,700,4000$ Oe, respectively. An uniaxial magnetic anisotropy constant and a saturation magnetization are $K_{1}=$ $0.4 \cdot 10^{5} \mathrm{erg} / \mathrm{cm}^{3}$ and $M_{s}=400 \mathrm{emu} / \mathrm{cm}^{3}$, respectively. A direction of A magnetic field is determined by the angles $\left(\theta_{H}=\pi / 2, \varphi_{H}=0\right)$. In Fig. 3 this direction is shown by an arrow. 
(a)

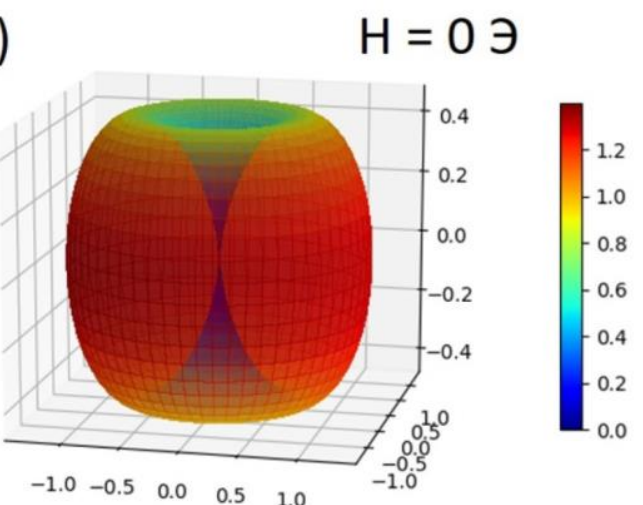

(b)

$H=300 \ni$
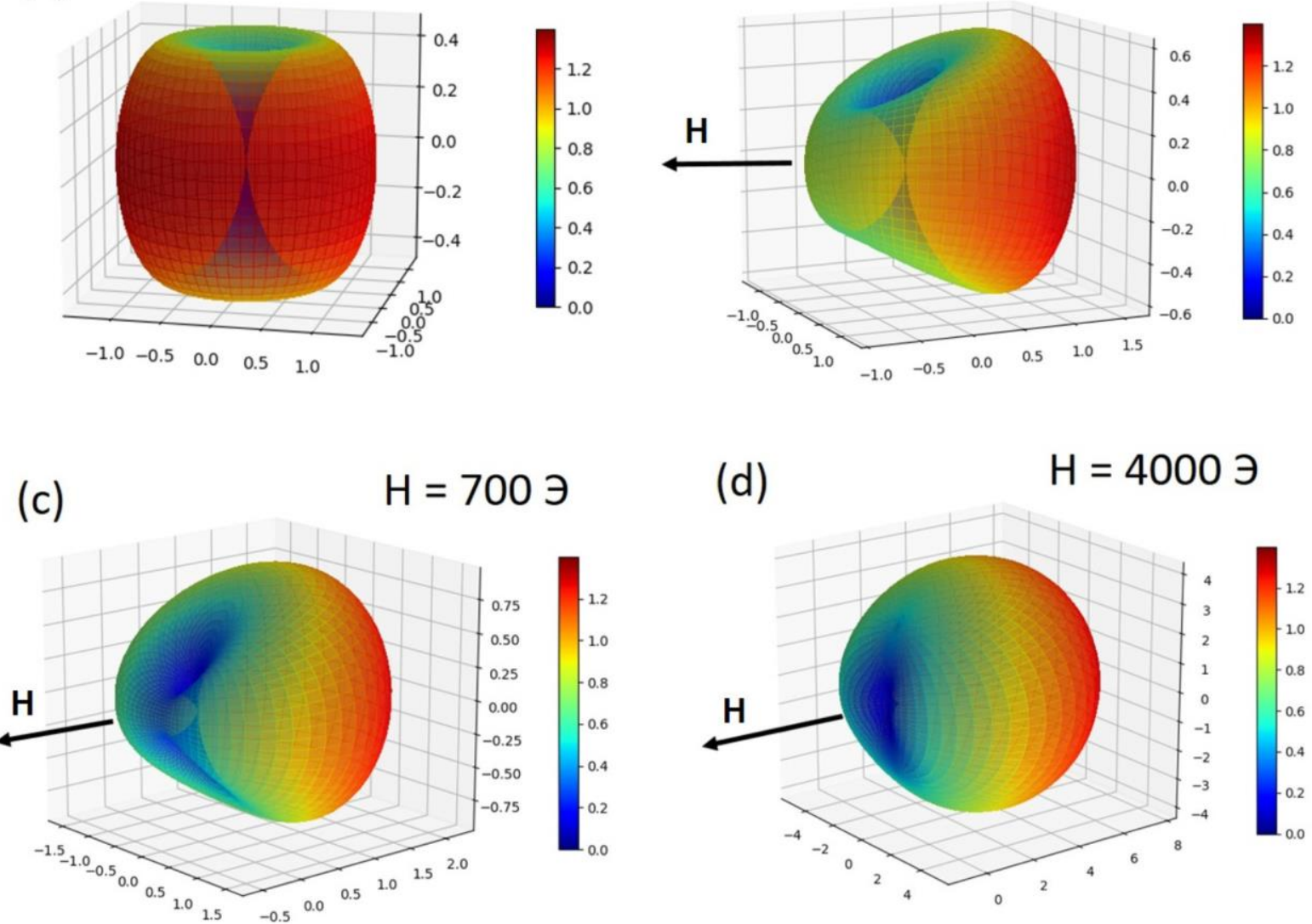

Fig. 3. Change in a total energy density of a uniaxial nanoparticle under an influence of an external magnetic field directed perpendicular to an easy axis of anisotropy.

As Fig. 3a - 3d show, with an increase of an external magnetic field $\boldsymbol{H}$, potential wells of a particle gradually merge, and in fields $\boldsymbol{H} \geq 2000$ Oe, only one potential well remains. Note that when constructing the energy surfaces shown in Fig. $3 \mathrm{~b}-3 \mathrm{~d}$, we add the corresponding positive constant to equation (6) to make the radius values positive. As indicated in the introduction, addition of an arbitrary constant to a total energy of a particle does not affect the physics of the processes, but leads to a corresponding change in the relative scales of the figures.

A similar picture of transformation of energy surfaces for nanoparticles with different types of magnetic anisotropy. For simplicity, only symmetric directions of an external magnetic field with respect to axes of a magnetic anisotropy of a nanoparticle are considered. In Fig. 4-7 show a conversion of a total energy density depending on a type of particle anisotropy and a magnitude of an applied external magnetic field. In each case, a direction of a magnetic field is indicated by an arrow.

Figure 4 shows an influence of an external field on a surface shape of a total energy density of a nanoparticle in a case of cubic anisotropy; an anisotropy energy density of this type is described by formula (2). Here, magnetic anisotropy constants and saturation magnetization are $K_{1 \mathrm{c}}=-0.4 \cdot 10^{5} \mathrm{erg} / \mathrm{cm}^{3}, K_{2 \mathrm{c}}=0, M_{s}=400 \mathrm{emu} / \mathrm{cm}^{3}$. 

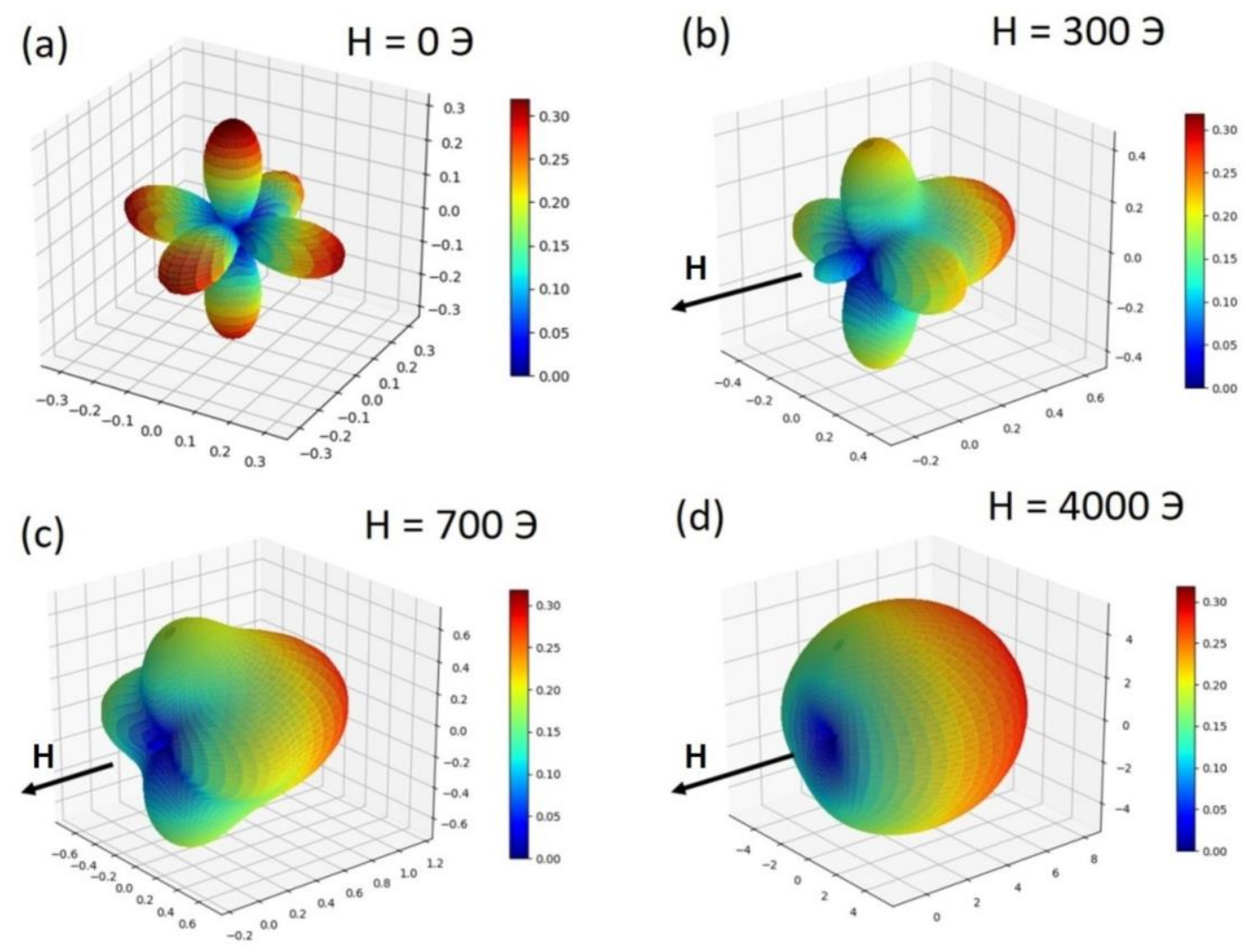

Fig. 4. An influence of an external magnetic field on a surface shape of a total energy density of a nanoparticle in a case of cubic magnetic anisotropy at $K_{1 \mathrm{c}}<0, K_{2 \mathrm{c}}=0$.

(Field direction $\theta_{H}=\pi / 2, \varphi_{H}=0$ )
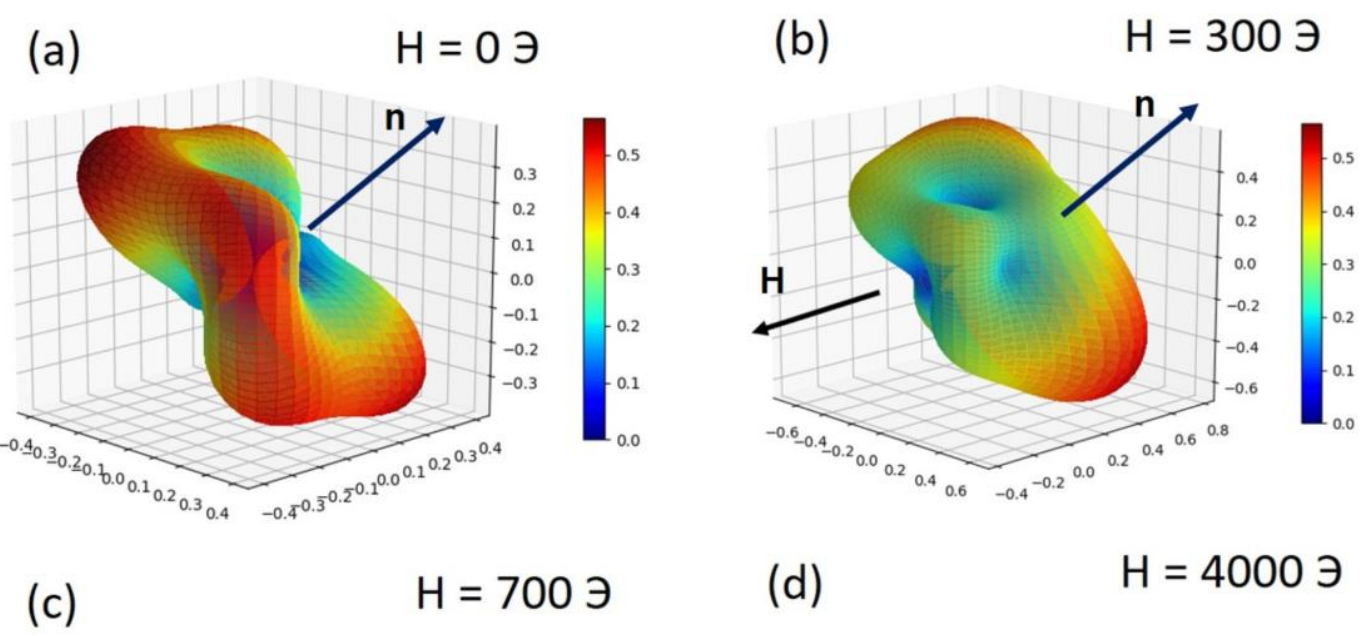
(d)

$$
H=4000 \ni
$$
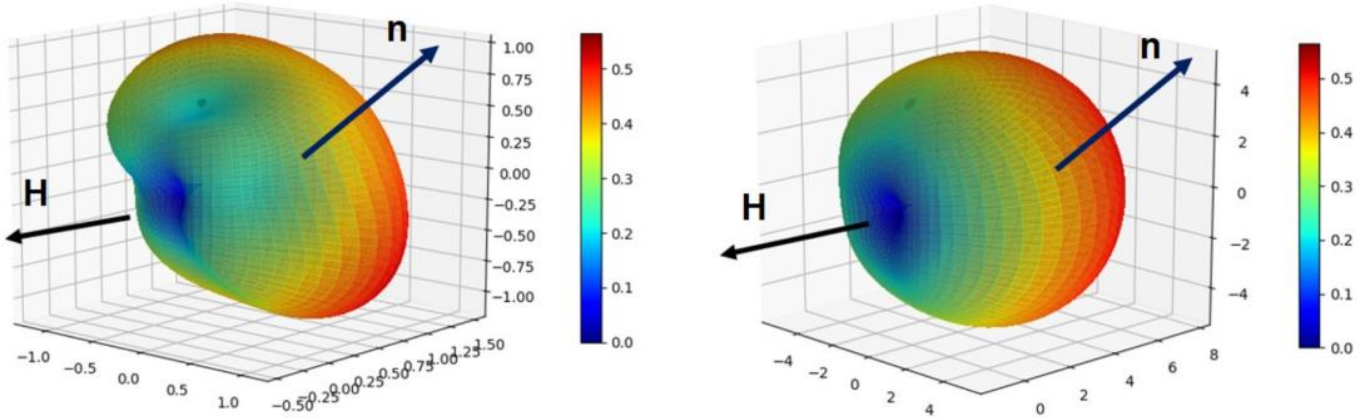

Fig. 5. An influence of an external magnetic field on a surface shape of a total energy density of a nanoparticle in a case of combined anisotropy (cubic anisotropy + uniaxial shape anisotropy) for $K_{1 \mathrm{c}}<0, K_{2 \mathrm{c}}=0$. (Field direction $\theta_{H}=\pi / 2, \varphi_{H}=0$ ) 
Figure 5 shows an influence of an external field on a surface shape of a nanoparticle total energy density in the case of combined anisotropy. Here, combined anisotropy is the sum of crystalline anisotropy, $w_{a}(\theta, \varphi) / K_{1 c}$, with shape anisotropy, $K_{e f}\left(1-(\vec{\alpha} \vec{n})^{2}\right) / K_{1 c}$. A uniaxial magnetic anisotropy constant and saturation magnetization are $K_{1 \mathrm{c}}=-0.4 \cdot 10^{5} \mathrm{erg} / \mathrm{cm}^{3}, K_{2 \mathrm{c}}=$ $\mathrm{o}, M_{s}=400 \mathrm{emu} / \mathrm{cm}^{3}$, a ratio of a shape anisotropy constant to a crystalline anisotropy constant is, $K_{e f} / K_{1 c}=0.1$. A direction of an easy shape anisotropy vector, $\boldsymbol{n}$, is indicated by an arrow.
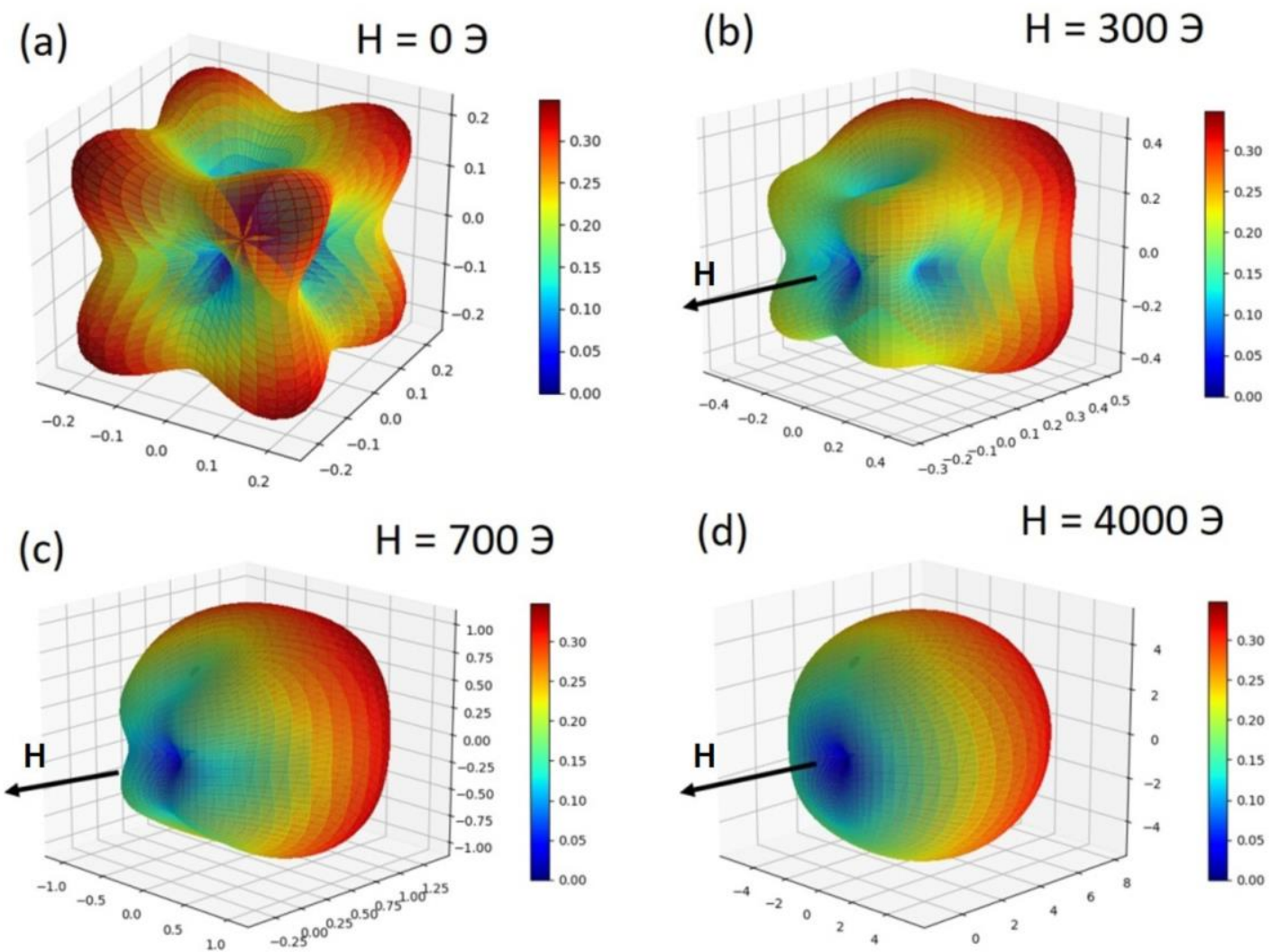

Fig. 6. An influence of an external magnetic field on a surface shape of a nanoparticle total energy density in a case of cubic anisotropy for $K_{1 \mathrm{c}}>\mathrm{O}, K_{2 \mathrm{c}}=\mathrm{O}$.

(Field direction $\theta_{H}=\pi / 2, \varphi_{H}=0$ )

Figure 6 shows a form of a reduced energy density of cubic magnetic anisotropy, $w_{a}(\theta, \varphi) / K_{1 c}$, equation (2), depending on a magnitude of an external field, for a case $K_{1 \mathrm{c}}>\mathrm{O}, K_{2 \mathrm{c}}=\mathrm{O}$. Constants of magnetic anisotropy and saturation magnetization are $K_{1 \mathrm{c}}=0.4 \cdot 10^{5} \mathrm{erg} / \mathrm{cm}^{3}, K_{2 \mathrm{c}}=0$, $M_{s}=400 \mathrm{emu} / \mathrm{cm}^{3}$.

Figure 7 shows a reduced energy density of uniaxial magnetic anisotropy (1) as a function of the external field for the case $K_{1}<0, K_{2}=0$. Constants of magnetic anisotropy and saturation magnetization are $K_{1}=-0.4 \cdot 10^{5} \mathrm{erg} / \mathrm{cm}^{3}, K_{2}=0, M_{s}=400 \mathrm{emu} / \mathrm{cm}^{3}$.

Figures 4-7 show that for large values of an applied external field, $\boldsymbol{H}>4000$ Oe, a complex geometry of a nanoparticle energy density, caused by a contribution of anisotropy, completely disappears. A single minimum of energy appears along the direction of the magnetic field. 

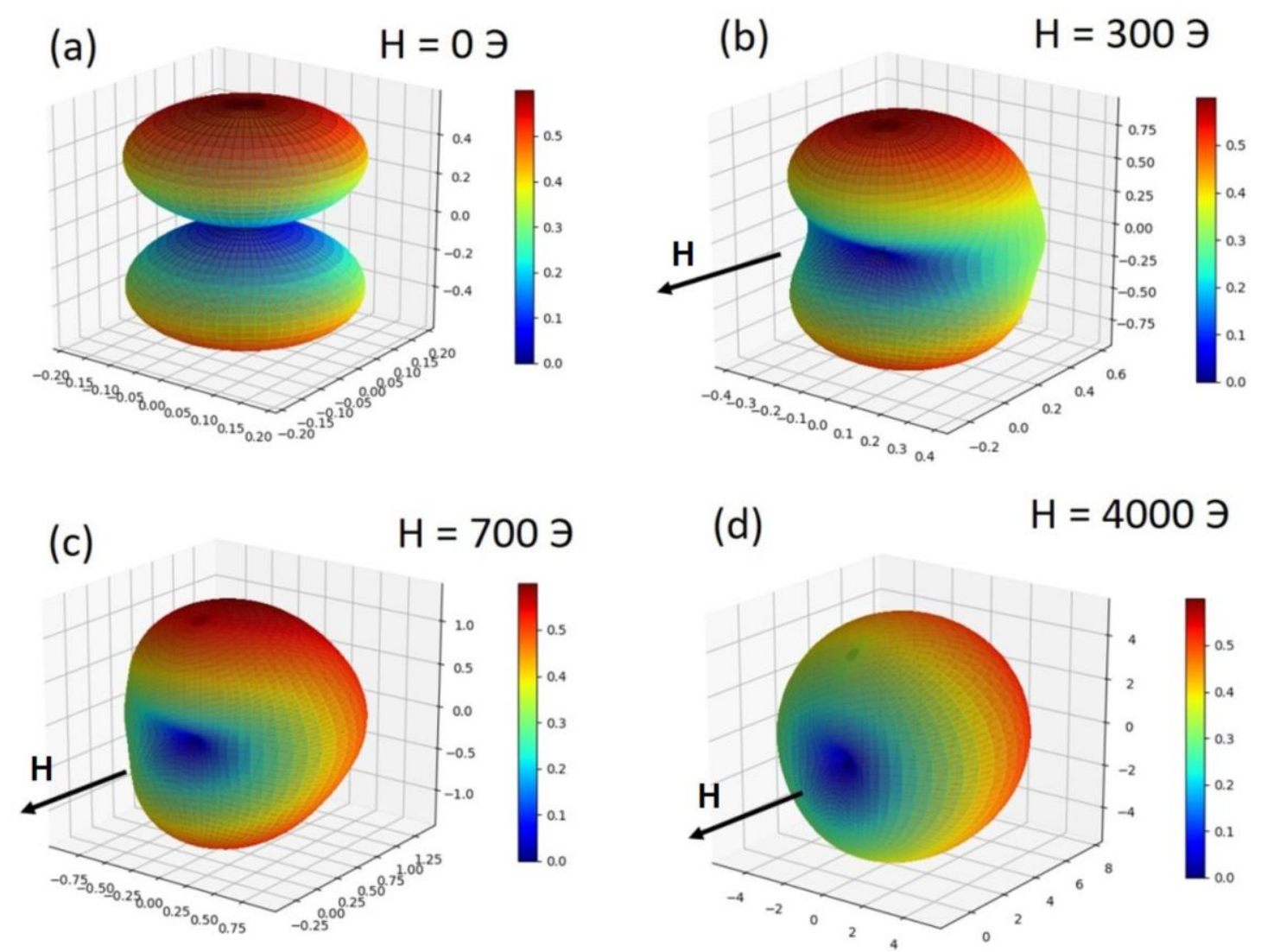

Fig. 7. An influence of an external magnetic field on a surface shape of a total energy density of a nanoparticle in a case of uniaxial anisotropy at $K_{1}<0, K_{2}=0$

(Field direction $\theta_{H}=\pi / 2, \varphi_{H}=0$ )

\section{Conclusion}

Thus, we see that properties of magnetic nanoparticles are determined by a whole set of geometric and magnetic parameters, namely, an external shape of particles, a distribution of easy axes of magnetocrystalline anisotropy, a value of magnetic anisotropy constants, and saturation magnetization. For applications, it is also very important whether a particle is singlecrystal or consists of individual crystallites of different spatial orientations connected by an exchange interaction. In real experimental ensembles, there is usually a significant scatter of nanoparticles in size and shape. Particles are often polycrystalline. In polycrystalline nanoparticles, anisotropic interactions are significantly averaged, which affects a characteristic size of a single domain and a coercive force of nanoparticles [16]. Therefore, magnetic properties of such ensembles of nanoparticles are very difficult to control.

\section{Acknowledgments}

The authors gratefully acknowledge the financial support of the Ministry of Education and Science of the Russian Federation in the framework of Increase Competitiveness Program of NUST “MISIS”, contract №K2A-2019-034.

\section{References}

1. S. Sun, C. B. Murray, D. Weller, L. Folks, A. Moser, Monodisperse FePt Nanoparticles and Ferromagnetic FePt Nanocrystal Superlattices // Science, Vol. 287, 2000, pp. 19891992.

2. López-Ortega, A., Lottini, E., Fernández, C. D. J., Sangregorio, C. Exploring the Magnetic Properties of Cobalt-Ferrite Nanoparticles for the Development of a Rare-Earth-Free Permanent Magnet // Chemistry of Materials, Vol. 27, 2015, pp. 4048-4056. 
3. Q.A. Pankhurst, N.K.T. Thanh, S.K. Jones, J. Dobson, Progress in applications of magnetic nanoparticles in biomedicine // J. Phys. D: Appl. Phys., Vol. 42, 2009, p. 224001

4. Dutz S., Hergt R., Magnetic nanoparticle heating and heat transfer on a microscale: Basic principles, realities and physical limitations of hyperthermia for tumour therapy // Int. J. Hyperthermia, Vol. 29, 2013, pp. 790-800.

5. Ortega D., Pankhurst Q.A., Magnetic hyperthermia // In O'Brien P editor Nanoscience: Nanostructures through Chemistry Royal Society of Chemistry: Cambridge, Vol. 1, 2013, pp. 60-88.

6. Périgo E.A., Hemery G., Sandre O., Ortega D., Garaio E., Plazaola F., Teran F.J., Fundamentals and advances in magnetic hyperthermia // Appl. Phys. Rev., Vol. 2, 2015, p. 041302.

7. S. Chikazumi, Physics of Magnetism. New York: Wiley, 1964.

8. W.F. Brown, Jr., Micromagnetics. New York - London: Wiley-Interscience, 1963.

9. A. Aharoni, Introduction to the Theory of Ferromagnetism. Oxford: Clarendon Press, 1996

10. L.D. Landau, E.M. Lifshitz, Electrodynamics of Continuous Media. New York: Pergamon, New York, 1984.

11. A. I. Akhiezer, V. G. Baryakhtar, and S. V. Peletminskii, Spin Waves. Amsterdam: NorthHolland, 1968.

12. V.V. Pilyugin, I. Millman. Visual Analytics and its Application in the Activities of the Laboratory "Scientific Visualization" MEPhI (2019). Scientific Visualization 11.5: 46 - 55, DOI: 10.26583/sv.11.5.05

13. Yu. S. Lagutin, A. A. Lagutina, F. V. Grigoriev, V. B. Sulimov, A. V. Tikhonravov (2018) Visualization of nanosize pores in the atomistic structure of thin films. Scientific Visualization 10.1: 128 - 134, DOI: 10.26583/sv.10.1.10

3. Translated with www.DeepL.com/Translator (free version)14. W. F. Brown, Jr. and A. H. Morrish, Effect of a Cavity on a Single-Domain Magnetic Particle // Phys. Rev., Vol. 105, 1957, p. 1198.

14. N.A. Usov, J.M. Barandiarán, Magnetic nanoparticles with combined anisotropy // J. Appl. Phys., Vol. 112, 2012, p. 053915.

15. V. A. Bautin, A. G. Seferyan, M. S. Nesmeyanov, N. A. Usov, Properties of polycrystalline nanoparticles with uniaxial and cubic types of magnetic anisotropy of individual grains // J. Magn. Magn. Mater., Vol. 460, 2018, p. 278. 